The beam quality parameter of spirally polarized beams

This article has been downloaded from IOPscience. Please scroll down to see the full text article.

2008 J. Opt. A: Pure Appl. Opt. 10125004

(http://iopscience.iop.org/1464-4258/10/12/125004)

View the table of contents for this issue, or go to the journal homepage for more

Download details:

IP Address: 147.96.14.16

The article was downloaded on 11/06/2013 at 16:53

Please note that terms and conditions apply. 


\title{
The beam quality parameter of spirally polarized beams
}

\author{
V Ramírez-Sánchez and G Piquero \\ Departamento de Óptica, Universidad Complutense de Madrid, 28040 Madrid, Spain \\ Received 23 July 2008, accepted for publication 10 October 2008 \\ Published 31 October 2008 \\ Online at stacks.iop.org/JOptA/10/125004
}

\begin{abstract}
Starting from the expression for the quality parameter of a superposition of two general fields, the case of beams that can be written in terms of the polarization basis introduced by Gori is investigated. Different types of this class of beam are studied and compared. In particular, spirally polarized beams are considered. As an example, polarized Bessel-Gauss beams are analyzed in detail.
\end{abstract}

Keywords: polarization, beam quality

\section{Introduction}

Optical beams with special polarization characteristics across the transverse section are a subject of increasing interest. Different kinds of non-uniformly polarized beams and methods for characterizing them have been proposed in the literature [1-7]. They allow us to improve several applications. In particular, radially and azimuthally polarized beams are used in optical trapping, material processing, particle acceleration, high resolution microscopy, etc [8-13]. These non-uniformly polarized beams can be considered as particular cases of the so-called spirally polarized beams (SPBs). A new basis for describing a class of paraxial non-uniformly polarized fields, including SPBs, has been introduced in the literature by Gori [14]. This kind of beam presents interesting distributions of the polarization across the beam profile that remain invariant upon free propagation. Following this proposal, different works studying the properties and characteristics of SPBs have been published $[15,16]$. Moreover, this polarization basis is useful for describing polarized beams with a single vortex of topological charge $m$. Beams carrying vortices are currently receiving much interest for their numerous applications, for example as optical tweezers.

On the other hand, in some applications in which beams with non-conventional distributions of polarization are used, a good beam quality is required. In some papers the beam quality factor of radially and azimuthally polarized beams before and after different optical systems is given [17-21], but only in a few papers are the theoretical analytical expressions derived [22]. To our knowledge the quality parameter of spirally polarized beams has not been calculated in the vectorial treatment yet. In our study we will consider the beam quality defined within the paraxial approach. Recent papers propose to extent the definition of the beam quality factor to non-paraxial vectorial beams [23, 24] but it is a subject that deserves further discussion.

The aim of this work is to study the paraxial beam quality parameter of beams that can be written in terms of the Gori basis, in particular spirally polarized fields. The paper is arranged as follows. In the next section we introduce the appropriate definitions and show the formalism to be used. We begin with the quality for the superposition of two general beams. The quality parameter for beams with rotationally symmetric irradiance and states of polarization given by the Gori polarization basis and their combinations is calculated in section 3. Different cases are investigated: (i) beams described by only one basis polarization state, (ii) beams synthesized by a combination of two orthogonal vectors of this basis, and (iii) beams synthesized by a combination of two nonorthogonal vectors of the same basis. As will be shown, these fields can be written as a superposition of vortex beams. The cases of integer and fractional vortex charges will be also studied. As an example, beams with Bessel-Gauss amplitude are analyzed in section 4. Finally, the main conclusions are derived in section 5 .

\section{Formalism and definitions}

Let us begin with a monochromatic field, E(r), propagating along the $z$-axis (assuming $z$ as the mean direction of the beam in the paraxial regime). We will consider that the beam waistwidth, $\omega_{0}$, is large enough compared to the wavelength, $\lambda$, i.e. $w_{0} / \lambda \gg 1$. In such a case the longitudinal component of the field is much smaller than the transversal components 
and can be neglected [15]. For totally polarized beams at plane $z=0$, the Jones vector in terms of the $s$-and $p$-components of the field is

$$
\mathbf{E}(\mathbf{r})=\left(E_{s}(\mathbf{r}), E_{p}(\mathbf{r})\right) .
$$

The second-order irradiance moments of a partially polarized and partially coherent beam are defined as [25-28]

$$
\begin{aligned}
& \langle\alpha \beta\rangle=\frac{1}{P} \iiint \alpha \beta \overline{\mathbf{E}^{\dagger}(\mathbf{r}+\mathbf{s} / 2, z) \mathbf{E}(\mathbf{r}-\mathbf{s} / 2, z)} \\
& \quad \times \exp (\mathrm{i} k \mathbf{s} \boldsymbol{\eta}) \mathrm{d} \mathbf{s} \mathrm{d} \mathbf{r} \mathrm{d} \eta,
\end{aligned}
$$

where the symbol $\dagger$ denotes the adjoint, the overbar represents an ensemble average, $k=2 \pi / \lambda$ with $\lambda$ the wavelength, and $P$ is the total power of the beam. In the above equation $\mathbf{r}=$ $\left(\mathbf{r}_{1}+\mathbf{r}_{2}\right) / 2$ and $\mathbf{s}=\left(\mathbf{r}_{1}-\mathbf{r}_{2}\right), \mathbf{r}_{1}$ and $\mathbf{r}_{2}$ being two points of the transverse section of the beam. On the other hand, $\boldsymbol{\eta}=(u, v)$, where $u$ and $v$ represent angles of propagation (without taking the evanescent waves into account) and $\alpha, \beta=x, y, u, v$.

In terms of these measurable moments the general expression of the paraxial beam quality parameter for polarized beams (in the waist plane) is given by (see for example [29])

$$
Q=\left\langle r^{2}\right\rangle\left\langle\eta^{2}\right\rangle
$$

As usual, $\left\langle r^{2}\right\rangle$ and $\left\langle\eta^{2}\right\rangle$ are connected with the minimum beam width and the far-field divergence, respectively. Note that $Q$ is related to the standard beam propagation factor $M^{2}$ by means of the expression $\left(M^{2}\right)^{2}=k^{2} Q$. Parameter $Q$ is invariant upon propagation through rotationally symmetric $\mathrm{ABCD}$ systems and gives us the focusing capability of a beam in near and far fields. The minimum value, $Q=1 / k^{2}$, is obtained for Gaussian beams. For the sake of brevity, in what follows it will be assumed that $\langle\mathbf{r}\rangle=\langle\boldsymbol{\eta}\rangle=0$ (this is simply equivalent to a shift of the coordinate system).

For totally coherent beams the previous irradiance moments in polar coordinates are calculated in a simple form as follows:

$$
\left\langle r^{2}\right\rangle=\frac{1}{P} \iint r^{2}|\mathbf{E}(r, \theta)|^{2} r \mathrm{~d} r \mathrm{~d} \theta,
$$

and

$$
\left\langle\eta^{2}\right\rangle=\frac{1}{k^{2} P} \iint\left(\left|\frac{\partial \mathbf{E}(r, \theta)}{\partial r}\right|^{2}+\frac{1}{r^{2}}\left|\frac{\partial \mathbf{E}(r, \theta)}{\partial \theta}\right|^{2}\right) r \mathrm{~d} r \mathrm{~d} \theta,
$$

with

$$
P=\iint|\mathbf{E}(r, \theta)|^{2} r \mathrm{~d} r \mathrm{~d} \theta
$$

Let us now consider a field that can be written as a superposition of two beams,

$$
\mathbf{E}(r, \theta)=\mathbf{E}_{1}(r, \theta)+\mathbf{E}_{2}(r, \theta) .
$$

By applying equations (3)-(5) to equation (7), the parameter $Q$ is given in terms of the beam quality of each beam, $\mathbf{E}_{1}$ and $\mathbf{E}_{2}$, in the form

$$
\begin{aligned}
Q= & \frac{P_{1}^{2}}{P^{2}} Q_{1}+\frac{P_{2}^{2}}{P^{2}} Q_{2}+\frac{P_{1} P_{2}}{P^{2}} Q_{\text {mix }} \\
& +\frac{P_{12}}{P}\left\langle r^{2}\right\rangle_{12}\left(\frac{P_{1}}{P}\left\langle\eta^{2}\right\rangle_{1}+\frac{P_{2}}{P}\left\langle\eta^{2}\right\rangle_{2}\right) \\
& +\frac{P_{12}}{P}\left\langle\eta^{2}\right\rangle_{12}\left(\frac{P_{1}}{P}\left\langle r^{2}\right\rangle_{1}+\frac{P_{2}}{P}\left\langle r^{2}\right\rangle_{2}\right) \\
& +\frac{P_{12}^{2}}{P^{2}}\left\langle r^{2}\right\rangle_{12}\left\langle\eta^{2}\right\rangle_{12},
\end{aligned}
$$

where

$$
\begin{gathered}
Q_{i}=\left\langle r^{2}\right\rangle_{i}\left\langle\eta^{2}\right\rangle_{i}, \\
Q_{\text {mix }}=\left\langle r^{2}\right\rangle_{1}\left\langle\eta^{2}\right\rangle_{2}+\left\langle r^{2}\right\rangle_{2}\left\langle\eta^{2}\right\rangle_{1}, \\
\left\langle r^{2}\right\rangle_{i j}=\frac{\iint r^{2}\left(\mathbf{E}_{i}^{*} \cdot \mathbf{E}_{j}\right) r \mathrm{~d} r \mathrm{~d} \theta}{\iint\left(\mathbf{E}_{i}^{*} \cdot \mathbf{E}_{j}\right) r \mathrm{~d} r \mathrm{~d} \theta}, \\
\left\langle\eta^{2}\right\rangle_{i j}=\frac{1}{k^{2}} \frac{\iint\left(\frac{\partial \mathbf{E}_{i}^{*}}{\partial r} \cdot \frac{\partial \mathbf{E}_{j}}{\partial r}\right)+\frac{1}{r^{2}}\left(\frac{\partial \mathbf{E}_{i}^{*}}{\partial \theta} \cdot \frac{\partial \mathbf{E}_{j}}{\partial \theta}\right) r \mathrm{~d} r \mathrm{~d} \theta}{\iint\left(\mathbf{E}_{i}^{*} \cdot \mathbf{E}_{j}\right) r \mathrm{~d} r \mathrm{~d} \theta}, \\
P_{i}=\iint\left(\mathbf{E}_{i}^{*} \cdot \mathbf{E}_{i}\right) r \mathrm{~d} r \mathrm{~d} \theta=\iint\left|\mathbf{E}_{i}(r, \theta)\right|^{2} r \mathrm{~d} r \mathrm{~d} \theta, \\
P_{12}=2 \iint\left(\mathbf{E}_{1}^{*} \cdot \mathbf{E}_{2}\right) r \mathrm{~d} r \mathrm{~d} \theta,
\end{gathered}
$$

with $i, j=1,2,\langle\alpha \beta\rangle_{i i}=\langle\alpha \beta\rangle_{i}, Q_{i}$ and $P_{i}$ being the beam quality parameter and the power of each field, $\mathbf{E}_{i}(r, \theta)$, and $P=P_{1}+P_{2}+P_{12}$ the total power of the beam. Note that the sign of the term $P_{12}$ will depend on the scalar product $\left(\mathbf{E}_{1}^{*} \cdot \mathbf{E}_{2}\right)$.

\section{Quality parameter of non-uniformly polarized beams generated with the Gori polarization basis}

In this section we will focus on beams of the form

$$
\mathbf{E}(\mathbf{r})=\mathbf{E}_{1}(r, \theta)+\mathbf{E}_{2}(r, \theta)=f(r) \hat{v}_{i}(\theta)+g(r) \hat{v}_{j}(\theta),
$$

where $i, j=1,2,3,4$ and $\hat{v}_{i}, \hat{v}_{j}$ are two different unitary vectors which depend only on the coordinate $\theta$. These vectors constitute the basis introduced by Gori [14] for describing a class of non-uniformly totally and linearly polarized beams. They are explicitly given by the following expressions:

$$
\begin{array}{ll}
\hat{v}_{1}(\theta)=\left(\begin{array}{c}
\cos m \theta \\
\sin m \theta
\end{array}\right), & \hat{v}_{2}(\theta)=\left(\begin{array}{c}
\cos m \theta \\
-\sin m \theta
\end{array}\right), \\
\hat{v}_{3}(\theta)=\left(\begin{array}{c}
\sin m \theta \\
-\cos m \theta
\end{array}\right), & \hat{v}_{4}(\theta)=\left(\begin{array}{c}
\sin m \theta \\
\cos m \theta
\end{array}\right),
\end{array}
$$

where $m$ is the charge of a single vortex. For $m=1$, $\hat{v}_{1}$ and $\hat{v}_{3}$ represent a radially or an azimuthally polarized beam, respectively. Vectors $\hat{v}_{2}$ and $\hat{v}_{4}$ represent other different non-uniformly and axially symmetric distributions of linear polarization. In particular, SPBs can be written as a combination of radially and azimuthally polarized beams (polarization maps at a transverse plane can be seen in [16]). For higher values of $m$ and different combinations of the vectors of this basis, more complicated distributions of the polarization across the beam profile are achieved. An 
interesting property of fields written as in equation (15) is that they keep their state of polarization during free propagation (assuming the paraxial approach).

Hereafter we refer to the set of vectors given by equation (16) as the polarization basis.

We can analyze three different possibilities:

(i) When $g(r)=0$, the field is written as

$$
\mathbf{E}(\mathbf{r})=f(r) \hat{v}_{i}(\theta),
$$

and the beam quality degrades proportionally to $m^{2}$. The analytical expression for $Q$ is

$$
Q=Q_{0}+\frac{m^{2}}{k^{2}}\left\langle r^{2}\right\rangle \frac{\int \frac{|f(r)|^{2}}{r^{2}} r \mathrm{~d} r}{\int|f(r)|^{2} r \mathrm{~d} r},
$$

where $Q_{0}$ is the beam quality for $m=0$ (uniformly totally polarized beam) that reads

$$
Q_{0}=\left\langle r^{2}\right\rangle\left\langle\eta^{2}\right\rangle
$$

with

$$
\begin{aligned}
\left\langle r^{2}\right\rangle & =\frac{\int r^{2}|f(r)|^{2} r \mathrm{~d} r}{\int|f(r)|^{2} r \mathrm{~d} r}, \\
\left\langle\eta^{2}\right\rangle & =\frac{1}{k^{2}} \frac{\int\left|\frac{\partial f(r)}{\partial r}\right|^{2} r \mathrm{~d} r}{\int|f(r)|^{2} r \mathrm{~d} r} .
\end{aligned}
$$

Equation (18) represents the quality parameter for a beam with state of polarization given by any vector of the polarization basis (equation (16)). In particular, for $m=$ 1 , equation (18) represents the quality for radially or azimuthally polarized beams.

It is interesting to note that the same quality is obtained for a uniformly polarized beam with a single vortex of charge $m$ written in the form

$$
\mathbf{E}(\mathbf{r})=f(r) \mathrm{e}^{\mathrm{i} m \theta} \hat{v},
$$

$\hat{v}$ being a normalized constant vector. The factor $\mathrm{e}^{\mathrm{i} m \theta}$ is the helical phase that characterizes a single vortex of charge $m$ located at $x=y=0$. In this case $Q_{0}$ represents the quality parameter of the beam without vortices (without the phase $\mathrm{e}^{\mathrm{i} m \theta}$ ) and the result is the same as in the scalar case [30]. As is mentioned in [30], the phase of the vortex produces an astigmatism in the field and it degrades the beam quality as $m$ increases.

(ii) If $\hat{v}_{i}(\theta) \cdot \hat{v}_{j}(\theta)=0$, the two polarization basis vectors are orthogonal and they must have the same charge $m$. Then, the beam quality parameter is given by equation (8) with $P_{12}=0$. Moreover, if $f(r)=g(r)$ the quality factor reduces to equation (18). SPBs are examples of this type.

Note that, for radially, azimuthally, and spirally polarized beams, the same value of $Q$ is obtained.

(iii) Now, the basis vectors are chosen in such a way that $\hat{v}_{i} \cdot \hat{v}_{j} \neq 0$ is satisfied as, for example,

$$
\hat{v}_{1}(\theta)=\left(\begin{array}{c}
\cos m \theta \\
\sin m \theta
\end{array}\right), \quad \hat{v}_{2}(\theta)=\left(\begin{array}{c}
\cos n \theta \\
-\sin n \theta
\end{array}\right) .
$$

Using Euler's formula we can write equation (15) in the form

$$
\mathbf{E}(\mathbf{r})=\frac{1}{2} A(r)\left(\begin{array}{c}
1 \\
-\mathrm{i}
\end{array}\right)+\frac{1}{2} A^{*}(r)\left(\begin{array}{l}
1 \\
\mathrm{i}
\end{array}\right)
$$

with

$$
A(r)=f(r) \mathrm{e}^{\mathrm{i} m \theta}+g(r) \mathrm{e}^{-\mathrm{i} n \theta} .
$$

Note that this non-uniformly polarized field can be expressed as a superposition of right and left circularly polarized beams with the same irradiance proportional to $|A(r)|^{2}$. The second-order moments are

$$
\left\langle r^{2}\right\rangle=\frac{P_{f}}{P}\left\langle r^{2}\right\rangle_{f}+\frac{P_{g}}{P}\left\langle r^{2}\right\rangle_{g}+\frac{P_{f g}}{P}\left\langle r^{2}\right\rangle_{f g},
$$

and

$$
\left\langle\eta^{2}\right\rangle=\frac{P_{f}}{P}\left\langle\eta^{2}\right\rangle_{f}+\frac{P_{g}}{P}\left\langle\eta^{2}\right\rangle_{g}+\frac{P_{f g}}{P}\left\langle\eta^{2}\right\rangle_{f g},
$$

where the subscripts $f$ and $g$ correspond to the functions $f(r)$ and $g(r)$, respectively, and

$$
\begin{aligned}
\left\langle r^{2}\right\rangle_{f} & =\frac{\int r^{2}|f(r)|^{2} r \mathrm{~d} r}{\int|f(r)|^{2} r \mathrm{~d} r}, \\
\left\langle r^{2}\right\rangle_{g} & =\frac{\int r^{2}|g(r)|^{2} r \mathrm{~d} r}{\int|g(r)|^{2} r \mathrm{~d} r}, \\
\left\langle r^{2}\right\rangle_{f g} & =\frac{\int r^{2} f(r) g(r) r \mathrm{~d} r}{\int f(r) g(r) r \mathrm{~d} r}, \\
\left\langle\eta^{2}\right\rangle_{f} & =\frac{1}{k^{2}} \frac{\int\left|\frac{\partial f(r)}{\partial r}\right|^{2} r \mathrm{~d} r+m^{2} \int \frac{|f(r)|^{2}}{r^{2}} r \mathrm{~d} r}{\int|f(r)|^{2} r \mathrm{~d} r}, \\
\left\langle\eta^{2}\right\rangle_{g} & =\frac{1}{k^{2}} \frac{\int\left|\frac{\partial g(r)}{\partial r}\right|^{2} r \mathrm{~d} r+n^{2} \int \frac{|g(r)|^{2}}{r^{2}} r \mathrm{~d} r}{\int|g(r)|^{2} r \mathrm{~d} r},
\end{aligned}
$$

$\left\langle\eta^{2}\right\rangle_{f g}=\frac{1}{k^{2}} \frac{\int \frac{\partial f(r)}{\partial r} \frac{\partial g(r)}{\partial r} r \mathrm{~d} r-m n \int \frac{1}{r^{2}} f(r) g(r) r \mathrm{~d} r}{\int f(r) g(r) r \mathrm{~d} r}$,

$$
P_{f}=(2 \pi) \int|f(r)|^{2} r \mathrm{~d} r
$$

$P_{g}=(2 \pi) \int|g(r)|^{2} r \mathrm{~d} r$,

$$
\begin{aligned}
& P_{f g}=2 \int f(r) g(r) r \mathrm{~d} r \int \cos [(m+n) \theta] \mathrm{d} \theta \\
& =2 \frac{\sin [2 \pi(m+n)]}{(m+n)} \int f(r) g(r) r \mathrm{~d} r .
\end{aligned}
$$

The beam quality is calculated by substituting the above moments in equation (8).

For integer values of $m+n$, the factor $P_{f g}=0$ due to the integral $\int \cos [(m+n) \theta] \mathrm{d} \theta$, which is only different from zero in the case of fractional orders of the charges.

For the particular situation $f(r)=g(r)$, the field is the superposition of two beams with identical amplitudes but with different vortex charges, resulting in the equalities $P_{f}=P_{g},\left\langle r^{2}\right\rangle_{f}=\left\langle r^{2}\right\rangle_{g}=\left\langle r^{2}\right\rangle_{f g}=\left\langle r^{2}\right\rangle$. This means that the beam widths and powers of each field are identical. 
On the other hand, the global divergence, $\left\langle\eta^{2}\right\rangle$, and $P_{f g}$, are

$$
\left\langle\eta^{2}\right\rangle=\left\langle\eta^{2}\right\rangle_{f}+\frac{t}{k^{2}} \frac{\int \frac{|f(r)|^{2}}{r^{2}} r \mathrm{~d} r}{\int|f(r)|^{2} r \mathrm{~d} r},
$$

and

$$
P_{f g}=2 P_{f} \operatorname{sinc}[2(m+n) \pi],
$$

with

$$
t=\frac{(m-n)^{2}+2 m n\{1-\operatorname{sinc}[2(m+n) \pi]\}}{2\{1+\operatorname{sinc}[2(m+n) \pi]\}} .
$$

Using equations (37)-(39), the beam quality results in being

$$
Q=Q_{0}+\frac{t}{k^{2}}\left\langle r^{2}\right\rangle \frac{\int \frac{|f(r)|^{2}}{r^{2}} r \mathrm{~d} r}{\int|f(r)|^{2} r \mathrm{~d} r} .
$$

Parameter $t$ satisfies $t \geqslant 0$. The minimum value, $t=0$, is reached for $m=n=0$, and the maximum, $t=$ $\left(m^{2}+n^{2}\right) / 2$, for $m+n=1,2,3, \ldots$.

Note that for beams with equal but opposite charges $n=-m$, the beam quality results in being as expressed in equation (18).

\section{Example: polarized Bessel-Gauss beams}

In order to illustrate the differences between the beam quality parameter in cases (i), (ii), and (iii) of section 3, we will consider a particular example. First of all, it should be noticed that the radial functions $f(r)$ and $g(r)$ must be chosen in such a way that the amplitudes vanish at the eye of the vortex in order to be single valued. For this reason we will study beams with Bessel-Gauss (BG) amplitude. These kinds of beams have the advantage that the spatial structure does not change under focusing or free propagation. Scalar zero-order Bessel-Gauss beams were proposed by Gori et al [31], and different authors have studied and experimentally synthesized Bessel-Gauss beams in the vectorial treatment [32-36]. Moreover, superposition of BG beams has been considered in the scalar treatment and there are several papers studying their propagation and spatial characteristics (see for example [37-39]).

In this section we consider a polarized beam of the form given in equation (15), with amplitudes

$$
f(r)=J_{m}(a r) \exp \left(-\frac{r^{2}}{w^{2}}\right),
$$

and

$$
g(r)=J_{n}(a r) \exp \left(-\frac{r^{2}}{w^{2}}\right)
$$

where $J_{m}(a r)$ is the Bessel function of order $m$, which coincides with the order of the vectors of the polarization basis, $a$ is a parameter that determines the transverse scale of the Bessel function, and $w$ is the width of the Gaussian factor.

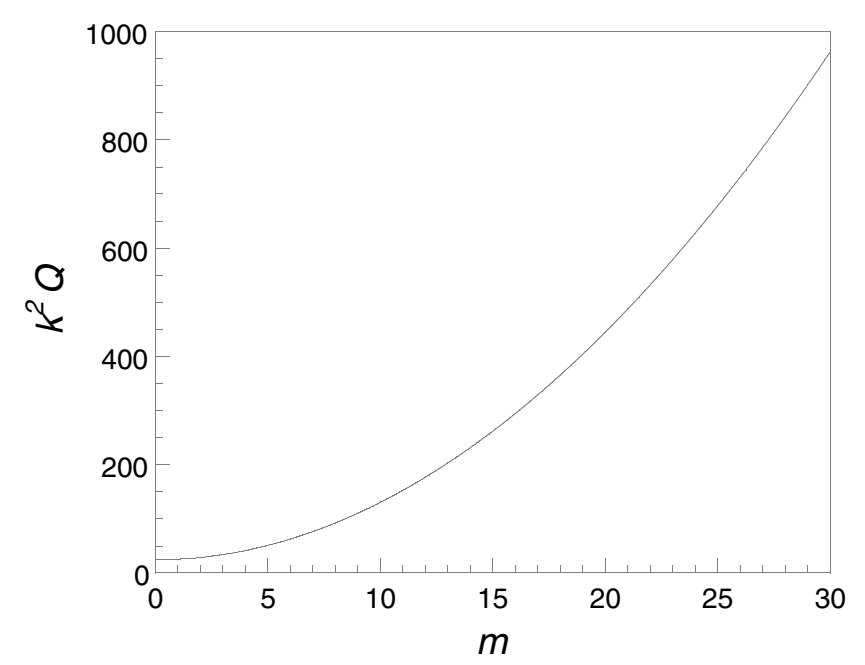

Figure 1. Normalized beam quality parameter $k^{2} Q$ versus the order $m$. The parameters of the Bessel-Gauss function are $a=9.92 \mathrm{~mm}^{-1}$ and $w=1 \mathrm{~mm}$.

We can analyze the three possibilities studied in the previous section, but now applied to BG beams:

(i) When $g(r)=0$, the beam quality parameter is the same as that of a vortex beam written as (see equation (22))

$$
\mathbf{E}(r, \theta)=J_{m}(a r) \exp \left(-\frac{r^{2}}{w^{2}}\right) \exp (\mathrm{i} m \theta) \hat{v},
$$

which represents a uniformly polarized beam. In this case $Q$, calculated from equation (18), coincides with the beam quality parameter for scalar Bessel-Gauss beams [39]. In figure 1 the normalized beam quality parameter $k^{2} Q$ has been plotted versus the order $m$. The beam quality degrades as $m$ increases. This behavior results from the fact that both the beam width and the beam divergence increase as $m$ grows (see [39]).

For radially or azimuthally polarized Bessel-Gauss beams the normalized quality parameter can be obtained from figure 1 for $m=1$, resulting in $k^{2} Q=25.7$.

(ii) For $\hat{v}_{i} \cdot \hat{v}_{j}=0, m$ should be equal to $n$, which implies that $f(r)$ is equal to $g(r)$, and therefore the behavior of the beam quality is the same as that in figure 1 .

(iii) When the two vectors $\hat{v}_{i}$ and $\hat{v}_{j}$ are not orthogonal, we can find two different cases:

(a) For integer values of $(n+m)$, the normalized beam quality parameter has been plotted versus the order $n$ for three different values of $m$ in figure 2. In this case, as $n$ increases the $Q$ parameter tends to the value given by equation (18) for fixed charge $m=1,3$, or 5. There is a value of $n$ for which the maximum degradation (lowest quality) of $Q$ is reached. When we change $n$, both the beam width and divergence have the same behavior as the beam quality. The maximum can be explained using figure 3 , in which we have plotted the irradiance distribution at the plane $z=0$, normalized to the value of the peak, across a diameter for three different beams with $m=5$ and 


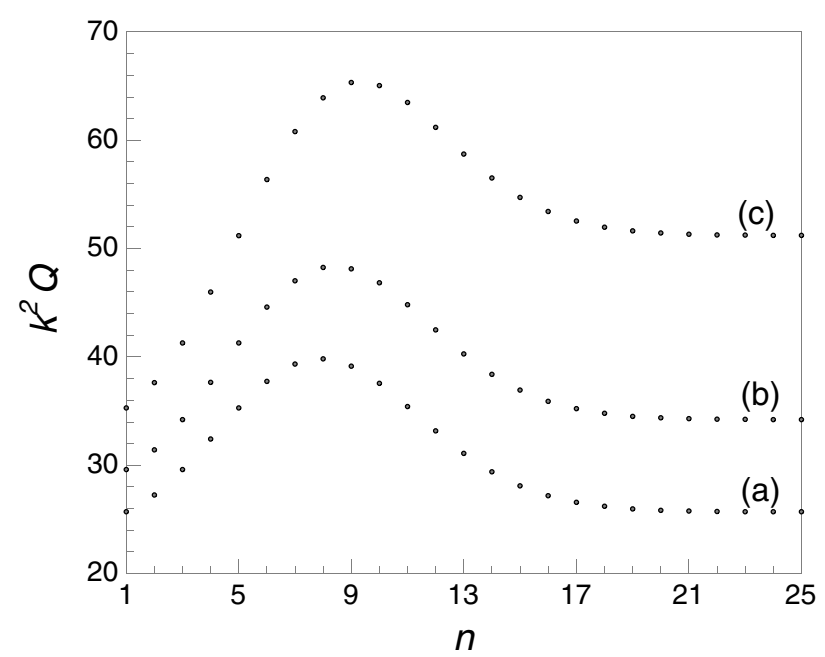

Figure 2. Normalized beam quality parameter $k^{2} Q$ versus the integer $n$ for (a) $m=1$, (b) $m=3$, and (c) $m=5$. The parameters $a$ and $w$ have the same values as in figure 1 .

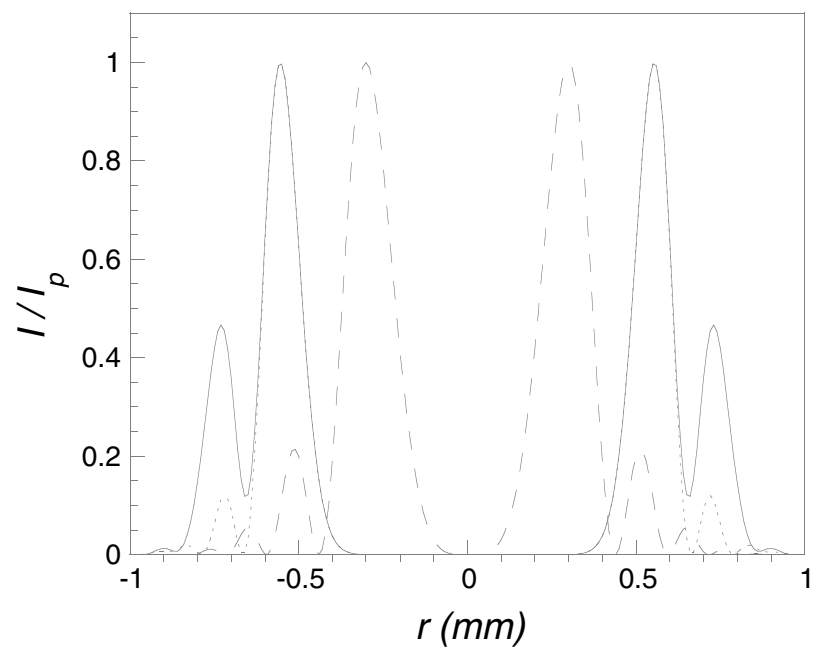

Figure 3. Irradiance beam profiles, normalized to the peak, across a diameter for $m=5$ and $n=1$ (dashed line), $n=10$ (solid line), and $n=15$ (dotted line). The parameters $a$ and $w$ have the same values as in figure 1.

$n=1,10$, and 15 . If we compare the irradiance profiles for these beams we can see that the secondary maxima are higher and situated farther away from the center of the beam for $n=10$. Those tails affect the second-order intensity beam moments, producing a degradation on the beam quality. In fact, as can be seen from figure 2 , the lowest quality is reached for $m=5$ and $n=10$.

An example of this type is the beam that constitutes the solution derived by Greene and Hall for describing beams generated by concentric-circlegrating surface emitting (CCGSE) semiconductor lasers $[2,3,34]$. Such a field can be written as

$$
\begin{aligned}
\mathbf{E}(\mathbf{r}) & =J_{l+1}(\text { ar }) \exp \left(-\frac{r^{2}}{w^{2}}\right) \hat{v}_{1}[(l+1) \theta] \\
& +J_{l-1}(\text { ar }) \exp \left(-\frac{r^{2}}{w^{2}}\right) \hat{v}_{2}[(l-1) \theta] .
\end{aligned}
$$

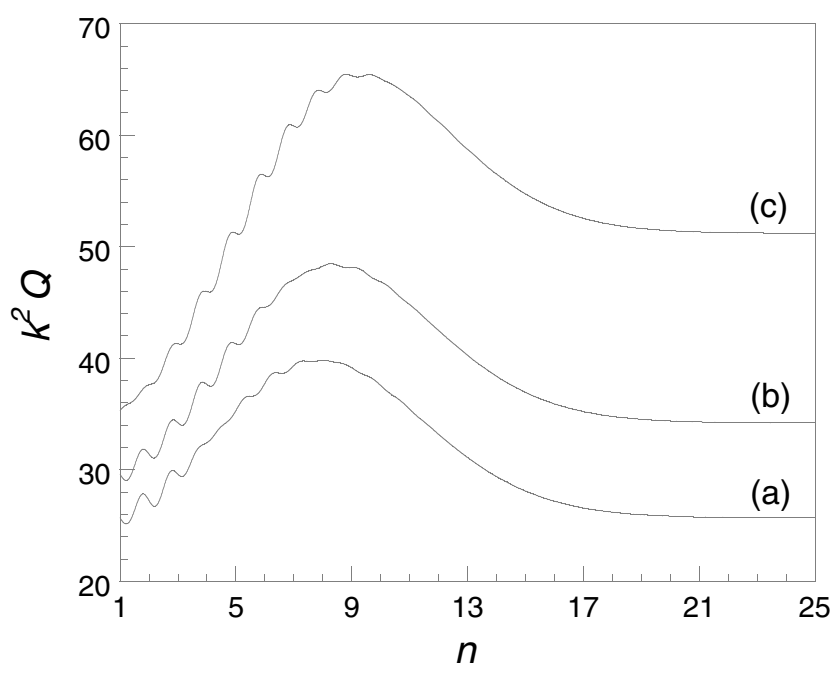

Figure 4. Normalized beam quality parameter $k^{2} Q$ versus the order $n$ (including the case of $(n+m)$ fractional) for (a) $m=1$,

(b) $m=3$, and (c) $m=5$. The parameters $a$ and $w$ have the same values as in figure 1 and 2 .

For these beams the quality parameter must be computed with $m=l+1$ and $n=l-1$. The values $k^{2} Q=29.6,41.3$, and 60.8 can be obtained directly from figure 2 for the integers $l=2,4$ and 6 .

(b) When $(n+m)$ is a fractional number we need to use equations (26)-(36) in equation (8) in order to obtain the quality parameter. Note that optical vortices with fractional charges have already been introduced in the literature [40-43]. In this case the factor sinc inside the parameter $t$ modulates the previous curves appearing ripples in the beam quality parameter (see figure 4).

\section{Conclusions}

We have investigated the paraxial quality parameter for beams whose state of polarization across the transverse plane can be written in terms of the Gori polarization basis. Particular examples are radially, azimuthally, and spirally polarized fields. Depending on the linear combinations of the basis states, the beam quality parameter changes in a different way. Furthermore, the same analytical expression for the quality parameter applies for the superposition of uniformly and totally polarized optical vortex beams. The effect of fractional vortex charges in the beam quality has also been considered. As a special case to illustrate these behaviors, polarized BesselGauss beams have been analyzed.

\section{Acknowledgments}

We thank Dr J C G de Sande and Dr J Serna for their valuable suggestions. This work has been supported by the Ministerio de Educación y Ciencia of Spain, project FIS2007-63396, and project CCG07-UCM/ESP-3070. 


\section{References}

[1] Tidwell S C, Ford D H and Kimura W D 1990 Generating radially polarized beams interferometrically Appl. Opt. 29 2234-9

[2] Erdogan T and Hall D G 1990 Circularly symmetric distributed feedback semiconductor laser: an analysis J. Appl. Phys. 68 1435-44

[3] Erdogan T, King O, Wicks G W, Hall D G, Anderson E and Rooks M J 1992 Circularly symmetric operation of a concentric-circle-grating surface emitting $\mathrm{AlGaAs} / \mathrm{GaAs}$ quantum well semiconductor laser Appl. Phys. Lett. 60 1921-3

[4] Tidwell S C, Kim G H and Kimura W D 1993 Efficient radially polarized laser beam generation with a double interferometer Appl. Opt. 32 5222-9

[5] Freund I 2001 Polarization flowers Opt. Commun. 199 47-63

[6] Mejías P M, Martínez-Herrero R, Piquero G and Movilla J M 2002 Parametric characterization of the spatial structure of non-uniformly polarized laser beams Prog. Quantum Electron. 26 65-130

[7] Martínez-Herrero R, Mejías P M, Piquero G and Ramírez-Sánchez V 2008 Global parameters for characterizing the radial and azimuthal polarization content of totally polarized beams Opt. Commun. 281 1976-80

[8] Zhan Q and Leger J R 2002 Focus shaping using cylindrical vector beams Opt. Express 10 324-31

[9] Zhan Q 2005 Trapping metallic Rayleigh particles with radial polarization Opt. Express 12 3377-82

[10] Niziev V G and Nesterov A V 1999 Influence of beam polarization on laser cutting efficiency J. Phys. D: Appl. Phys. 32 1455-61

[11] Nesterov A V and Niziev V G 2000 Laser beams with axially symmetric polarization J. Phys. D: Appl. Phys. 33 1817-22

[12] Liu Y, Cline D and He P 1999 Vacuum laser acceleration using a radially polarized $\mathrm{CO}_{2}$ laser beam $\mathrm{Nucl}$. Instrum. Methods Phys. Res. A 424 296-303

[13] Novotny L, Beversluis M R, Youngworth K S and Brown T G 2001 Longitudinal field modes probed by single molecules Phys. Rev. Lett. 86 5251-4

[14] Gori F 2001 Polarization basis for vortex beams J. Opt. Soc. Am. A 18 1612-7

[15] Borghi R and Santarsiero M 2004 Nonparaxial propagation of spirally polarized optical beams J. Opt. Soc. Am. A 21 2029-37

[16] Borghi R, Santarsiero M and Alonso M A 2005 Highly focused spirally polarized beams J. Opt. Soc. Am. A 22 1420-31

[17] Moshe I, Jackel S and Meir A 2003 Production of radially or azimuthally polarized beams in solid-state lasers and the elimination of thermally induced birefringence effects Opt. Lett. 28 807-9

[18] Roth M S, Wyss E W, Glur H and Weber H P 2005 Generation of radially polarized beams in a Nd:YAG laser with self-adaptative overcompensation of the thermal lens Opt. Lett. 30 1665-7

[19] Machavariani G, Lumer Y, Moshe I, Meir A and Jackel S 2007 Efficient extracavity generation of radially and azimuthally polarized beams Opt. Lett. 32 1468-70

[20] Moshe I, Jackel S, Meir A, Lumer Y and Leibush E 2007 $2 \mathrm{~kW}, M^{2}<10$ radially polarized beams from aberration-compensated rod-based Nd:YAG lasers Opt. Lett. 32 47-9

[21] Machavariani G, Lumer Y, Moshe I, Meir A and Jackel S 2008 Spatially-variable retardation plate for efficient generation of radially- and azimuthally-polarized beams Opt. Commun. $281732-8$

[22] Martínez-Herrero R, Piquero G and Mejías P M 2008 Beam quality changes of radially and azimuthally polarized fields propagating through quartic phase plates Opt. Commun. 281 756-9

[23] Kang X and Lü B 2005 The $\mathbf{M}^{2}$ factor of nonparaxial Hermite-Gaussian beams and related problems Optik $116232-6$

[24] Kang X, He Z and Lü B 2007 Far-field properties and beam quality of vectorial Hermite-Laguerre-Gaussian beams beyond the paraxial approximation Opt. Laser Technol. 39 1046-53

[25] Lavi S, Prochaska R and Keren E 1988 Generalized beam parameters and transformation law for partially coherent light Appl. Opt. 27 3696-703

[26] Bastiaans M J 1989 Propagation laws for the second-order moments of the Wigner distribution function in first-order optical systems Optik 82 173-81

[27] Siegman A E 1990 New developments in laser resonators Proc. SPIE 1224 2-14

[28] Serna J, Martínez-Herrero R and Mejías P M 1991 Parametric characterization of general partially coherent beams propagating through ABCD optical systems J. Opt. Soc. Am. A 8 1094-8

[29] Lü Q, Dong S and Weber H 1995 Analysis of TEM Do laser beam degradation caused by a birefringent Nd:YAG rod Opt. Quantum Electron. 27 777-83

[30] Ramee S and Simon R 2000 Effect of holes and vortices on beam quality J. Opt. Soc. Am. A 17 84-94

[31] Gori F, Guattari G and Padovani C 1987 Bessel Gauss beams Opt. Commun. 64 491-5

[32] Jordan R H and Hall D G 1994 Free space azimuthal paraxial wave equation: the azimuthal Bessel-Gauss beam solution Opt. Lett. 19 427-9

[33] Greene P L and Hall D G 1996 Diffraction characteristics of the azimuthal Bessel-Gauss beam J. Opt. Soc. Am. A 13 962-6

[34] Greene P L and Hall D G 1998 Properties and diffraction of vector Bessel-Gauss beams J. Opt. Soc. Am. A 15 3020-7

[35] Novitsky A V and Novitsky D V 2007 Negative propagation of vector Bessel beams J. Opt. Soc. Am. A $242844-9$

[36] Seshadri S R 2008 Electromagnetic modified Bessel-Gauss beams and waves J. Opt. Soc. Am. A $251-8$

[37] Orlov S and Stabinis A 2004 Propagation of superpositions of coaxial optical Bessel beams carrying vortices J. Opt. A. Pure Appl. Opt. 6 S259-62

[38] Tao S H, Yuan X C, Lin J and Burge R E 2005 Residue orbital angular momentum in interferenced double vortex beams with unequal topological charges Opt. Express 14 535-41

[39] Borghi R and Santarsiero M 1997 M $^{2}$ factor of Bessel-Gauss beams Opt. Lett. 22 262-4

[40] Berry M V 2004 Optical vortices evolving from helicoidal integer and fractional phase steps J. Opt. A: Pure Appl. Opt. 6 259-68

[41] Basistiy I V, Pasko V A, Slyusa V V, Soskin M S and Vasnetsov M V 2004 Synthesis and analysis of optical vortices with fractional topological charges J. Opt. A: Pure Appl. Opt. 6 S166-9

[42] Leach J, Yao E and Padgett M J 2004 Observation of the vortex structure of a non-integer vortex beam New J. Phys. 671

[43] Tao S H and Yuan X C 2005 Fractional optical vortex beam induced rotation of particles Opt. Express 13 7726-31 